\title{
Similar Clinical Outcomes in Patients with Systemic Juvenile Idiopathic Arthritis and Adult-Onset Still's Disease Treated with Canakinumab: Bayesian and Population Model-Based Analyses
}

\author{
Cornelia Dunger-Baldauf (iD · Priscila Nakasato · Stephanie Noviello •
}

Sarah Whelan · Bruno Bieth

Received: November 8, 2021 / Accepted: December 22, 2021 / Published online: January 19, 2022

(c) The Author(s) 2022

\section{ABSTRACT}

Introduction: Systemic juvenile idiopathic arthritis (sJIA) and adult-onset Still's disease (AOSD) represent pediatric and adult variants of the Still's disease continuum. To determine whether clinical outcomes between patients with sJIA and AOSD were similar, Bayesian and population model-based analyses were conducted on endpoints from studies of canakinumab in both patient populations. The objective was to further support the efficacy of canakinumab in patients with AOSD.

Methods: A Bayesian analysis of endpoints from a study of canakinumab in AOSD was conducted borrowing information from five pooled sJIA studies using a robust meta-analytic predictive (MAP) approach. Similarity of clinical outcomes across populations was fulfilled if the

Supplementary Information The online version contains supplementary material available at https:// doi.org/10.1007/s40744-021-00422-9.

C. Dunger-Baldauf $(\varangle) \cdot$ B. Bieth

Novartis Pharma AG, Postfach, 4002 Basel, Switzerland

e-mail: cornelia.dunger-baldauf@novartis.com

P. Nakasato $\cdot$ S. Noviello

Novartis Pharmaceuticals Corporation, East

Hanover, NJ, USA

S. Whelan

Novartis Pharma AG, Dublin, Ireland
AOSD study posterior median fell within the 95\% predicted credible interval for the outcome of interest, based on the pooled sJIA data. Population model-based analyses (pharmacokinetic $[\mathrm{PK}]$ and PK/pharmacodynamic [PKPD]) were conducted to compare the pharmacokinetics and exposure-response relationships between populations.

Results: The AOSD study posterior medians for adapted American College of Rheumatology (ACR)30 response, continuous adapted ACR response, number of active joints, C-reactive protein, and absence of fever were within the 95\% credible interval for the prediction of the MAP analysis from the pooled sJIA data, supporting the similarity in outcomes between patient populations. PK analysis demonstrated comparable exposure across sJIA age groups and patients with AOSD. PKPD relationships were consistent across patient populations. Analyses indicated that no therapeutic benefit can be expected from a dose increase in patients with AOSD.

Conclusion: The analyses presented support the similarity of clinical outcomes following treatment with canakinumab in patients with sJIA and AOSD.

Keywords: Adult-onset Still's disease; Bayes theorem; Canakinumab; Systemic juvenile idiopathic arthritis 


\section{Key Summary Points}

Why carry out this study?

Systemic juvenile idiopathic arthritis (sJIA) and adult-onset Still's disease (AOSD) represent pediatric and adult variants, respectively, of the Still's disease continuum.

Canakinumab has been shown to be effective at achieving rapid and sustained efficacy in multiple sJIA clinical trials, with positive trends also observed, in a small phase II, investigator-initiated, randomized, placebo-controlled trial in patients with AOSD.

In these analyses, Bayesian inference was used to support the similar efficacy of canakinumab in patients with AOSD borrowing data from the pediatric population.

\section{What was learned from the study?}

The analyses described in this publication support the similarity of clinical outcomes following treatment with canakinumab across patients with AOSD or sJIA.

Despite limited clinical data for canakinumab in patients with AOSD, the analyses presented in this publication contributed towards US regulatory approval of canakinumab for the treatment of AOSD in 2020, making it the only US Food and Drug Administrationapproved drug for the treatment of Still's disease (including both sJIA and AOSD).

\section{INTRODUCTION}

Systemic juvenile idiopathic arthritis (sJIA) and adult-onset Still's disease (AOSD) are rare autoinflammatory diseases that share similar pathogenesis along with many clinical, genetic, and laboratory features [1-3]. Based on their similarities, sJIA (onset $<16$ years of age) and AOSD (onset $\geq 16$ years of age) are considered to represent pediatric and adult variants, respectively, of the Still's disease continuum with different ages of onset [1-3]. This is further supported by similar biomarker profiles, including cytokine and transcriptomic data [4]. In addition to the classic triad of spiking fever, skin rash, and chronic arthritis/arthralgia, sJIA and AOSD are both associated with macrophage activation syndrome, a potentially life-threatening complication [1-3].

Based on the observed activation of the innate immune system and elevated levels of inflammatory cytokines in Still's disease, interleukin (IL)-1 inhibition has been highlighted as an effective treatment strategy [1, 3]. In an analysis of 27 studies of patients with Still's disease treated with anakinra, an IL-1 receptor antagonist, patients were able to achieve a clinically meaningful response to treatment (American College of Rheumatology 50\% [ACR50] response or clinically inactive disease) and taper the use of corticosteroids [5]. Furthermore, an analysis of pooled data from patients with sJIA $(N=301)$ indicated that older adolescent/young adult (age $\geq 16$ years) and adolescent (age 12 to $<16$ years) patients show similar clinical responses to canakinumab treatment, an IL-1 $\beta$ antagonist, when compared with pediatric patients (aged 2 to $<12$ years), suggesting treatment with canakinumab may be effective across both pediatric and adult populations [2].

Canakinumab has been shown to be effective at achieving rapid and sustained efficacy in multiple sJIA clinical trials [6-9]. However, the limited number of randomized controlled studies conducted in patients with AOSD makes drawing definitive conclusions on the efficacy of IL-1 inhibition in AOSD challenging [4, 5]. CONSIDER was a phase II, investigator-initiated, multicenter, double-blind, placebo-controlled study investigating the efficacy and safety of canakinumab in patients with AOSD and active joint involvement (tender and swollen joint counts of $\geq 4$ each) using the approved dosing regimen for sJIA. Treatment with canakinumab $(n=18)$ clearly led to more favorable results than with placebo $(n=17)$ in 
several outcome measures, including Disease Activity Score 28 joint count using erythrocyte sedimentation rate/C-reactive protein (DAS28$\mathrm{ESR} / \mathrm{CRP}$ ) and ACR response rates, although the study was terminated prematurely (due to the rare nature of AOSD and challenges with enrollment) and the primary endpoint was not met [4].

Bayesian analysis is a statistical method that utilizes prior information derived from sources in a specific population to update the evidence for another group [10]. This method is specifically useful when interpreting results from clinical trials with low patient numbers, such as those involving patients with rare diseases, if relevant data from external sources are available. Bayesian statistics have been previously utilized to borrow data from adult clinical trials as evidence for pediatric treatment response [10]. Reports from expansions of drug labels to include the pediatric population (e.g., infliximab in pediatric ulcerative colitis) have been used to demonstrate how Bayesian statistics can provide more precise inference on the treatment response in the pediatric population [10]. Interest in this type of statistical analysis to inform regulatory decisions is ever increasing [11].

Population model-based analyses include population pharmacokinetic (PK) and population PK/pharmacodynamic (PKPD) modeling. Population PK modeling is used to establish a relationship between treatment dose and blood concentration in various populations by characterizing intra- and inter-individual variability, providing a basis for possible adjustment among different subgroups [12]. Population PKPD modeling incorporates the relationship between blood concentration of a drug and pharmacodynamic responses, exploring possible differences in exposure-response among population subsets [12].

With a larger dataset being available from patients with sJIA than for AOSD, and the acceptance that SJIA and AOSD form the Still's disease continuum [1-3], Bayesian analysis and population model-based analyses were considered to be a clinically meaningful approach to investigate similarity in clinical outcomes. Therefore, both approaches were conducted on endpoints from studies of canakinumab in sJIA and AOSD to assess the similarity in clinical outcomes between patient populations. Population model-based analyses were also conducted to support the similarity in exposure and exposure-response between patient populations.

\section{METHODS}

The sJIA studies included in the Bayesian analysis were required to have been conducted in phase III trials and completed at the time the analysis was planned. In addition, the sJIA studies were required to have a similar canakinumab dosing regimen alongside similar efficacy endpoints up to week 12 compared with the AOSD study. Due to the rarity of the disease, only a limited number of phase III studies investigating the use of canakinumab in patients with sJIA have been conducted; data available up to week 12 of these studies met the requirements and were included in the analysis. For an overview of the studies used, see Electronic Supplementary Material (ESM) Table 1. A description of the methods and ethics approvals for the studies outlined have been detailed previously in their respective publications $[4,6-9,13]$.

This article is based on previously conducted studies and does not contain any new studies with human participants or animals performed by any of the authors.

\section{Bayesian Analysis}

A Bayesian analysis of endpoints from the AOSD study was conducted borrowing information from the sJIA studies (ESM Table 1). Full analysis set (FAS) populations from the sJIA studies (defined in the individual studies) and from the AOSD study (randomized patients with AOSD and high baseline severity who received at least 1 dose of canakinumab from baseline to week 12) were used. The primary time point considered for the analyses was week 12/day 85 , as week 12 was the primary analysis time point for the AOSD study. 
A robust meta-analytic predictive (MAP) analysis was used (ESM Methods file) as previously described [14]. This Bayesian approach protects against an inappropriate extrapolation in case of discrepancies between sJIA and AOSD data. The posterior distribution for each variable was obtained based on the robust MAP prior (sJIA pooled data) and AOSD study data. Similarity of clinical outcomes across the two populations was considered to be fulfilled if the AOSD study posterior median fell within the 95\% predicted credible interval for the outcome of interest, based on the prediction of the MAP from the sJIA pooled data.

\section{Outcomes of Interest}

Variables common to the AOSD and sJIA studies considered for analysis included the adapted ACR30 response, continuous adapted ACR response, number of active joints, serum CRP levels, and absence of fever. Where variables were not identical, such as functional ability assessed by the Childhood Health Assessment Questionnaire and the Health Assessment Questionnaire Disability Index, they were not included in the Bayesian inference of single parameters but were used in the calculation of the adapted ACR response.

As the sJIA and AOSD studies were conducted over different periods of time, without the intent of having the AOSD study replicate the results from the sJIA studies, there were differences in entry criteria and data collection. Therefore, an assessment of the variations between the studies was conducted and the efficacy parameters harmonized between sJIA and AOSD studies. As data on limitation of movement (LoM) were collected differently in the sJIA and AOSD studies for the adapted ACRbased endpoints and active joints, this criterion was not used in the definition of the harmonized variables. Details on how the adapted ACR response (regardless of LoM) was defined are provided in ESM Table 2. Endpoints included the adapted ACR30 score, the adapted continuous ACR score, and active joints count. The adapted ACR30 response was defined as $\geq 30 \%$ reduction between baseline and post- baseline values in $\geq 3$ of the following five parameters, with no more than one variable worsening by $>30 \%$ : number of active joints excluding LoM assessment; Physician Assessment of Disease Activity; Patient's/Parent's Assessment of Disease Activity; functional ability; CRP; absence of intermittent fever in the preceding week (final criterion was required to achieve an adapted ACR response).

In the case of missing parameters, if the values of the available parameters were fully conclusive, an adapted ACR30 response was determined, otherwise non-response was imputed. Continuous ACR response was defined as the highest level for which the patient was an ACR responder.

As data on rash were collected differently in the sJIA and AOSD studies (collected in patient's daily symptoms diary or physical exam in the sJIA studies and as an adverse event in the AOSD study), it was not possible to perform the robust MAP approach for this variable.

\section{Population Model-Based Analyses}

Exposure (population PK) and exposure-response (population PKPD) relationships were compared between the sJIA and AOSD populations at the canakinumab dose $4 \mathrm{mg} / \mathrm{kg}$ up to a maximum of $300 \mathrm{mg}$ every 4 weeks (Q4W) subcutaneously (s.c.).

To explore the age effect on PK and PKPD relationships across patient populations, data from the sJIA studies were pooled and compared across different age groups (children [2 to $<12$ years], adolescents [ 12 to $<16$ years] and older adolescents/young adults [ $\geq 16$ years]; age groups reflect age at study entry, not onset of sJIA). The pooled sJIA data were then compared to data from patients with AOSD in the age range of $22-70$ years. Analyses were performed using data from the first 12 weeks of treatment, as this was the longest duration for which all patients had data available on either canakinumab or placebo treatment. Canakinumab concentrations were analyzed using a population pharmacokinetic model previously developed for sJIA [15].

Exposure-response data were compared for continuous adapted ACR response, adapted 
ACR30 response, and number of active joints. CRP, DAS28-CRP, and absence of fever were also compared (ESM Methods file). Although DAS28ESR based response was the primary endpoint for the AOSD study, ESR and subsequently DAS28-ESR were not measured in the sJIA studies. Therefore, data were compared for both CRP and DAS28-CRP. Since efficacy data were comparable at week 8 and 12 for all studies (sJIA and AOSD), the two visits were pooled to make best use of available data. To assess the PKPD benefit of increasing the dose of canakinumab with respect to DAS28-CRP in patients with AOSD, an Emax model was fitted to DAS28-CRP change from baseline, using combined data at weeks 8 and 12 .

\section{RESULTS}

\section{Bayesian Analysis}

\section{Adapted ACR30 Response at Week 12}

In both the sJIA and AOSD studies, a rapid response to canakinumab treatment was observed at week 12 (ESM Table 3). The AOSD study posterior median was within the 95\% credible interval for the prediction of the MAP from the pooled sJIA data, supporting the similarity in outcomes between the patient populations (Fig. 1A).

\section{Reduction in the Number of Active Joints from Baseline at Week 12}

In both the sJIA and AOSD studies, the median number of active joints decreased during the initial 12 weeks of treatment (ESM Table 3). The AOSD study posterior median was within the 95\% credible interval for the prediction of the MAP from the pooled sJIA data, which supported the similarity in response to canakinumab treatment across populations (Fig. 1b).

\section{CRP Level at Week 12}

Median baseline values for CRP were higher in the sJIA studies compared with the AOSD study (124.5-132.8 vs. $38.0 \mathrm{mg} / \mathrm{L}$, respectively), which was most likely due to differences in the inclusion criteria. Consequently, the potential for reduction in mean CRP was higher for the sJIA population. Population differences for this clinical outcome would have been difficult to interpret. CRP level at week 12 was therefore analyzed.

In both the AOSD and SJIA populations, median CRP levels at week 12 were $<10 \mathrm{mg} / \mathrm{L}$ (ESMTable 3); the AOSD study posterior median was within the $95 \%$ credible interval for the prediction of the MAP from the pooled sJIA data, supporting the similarity in response to canakinumab treatment across populations (Fig. 1c).

\section{Continuous Adapted ACR and Absence of Fever at Week 12}

Continuous adapted ACR (ESM Fig. 1) and absence of fever (Fig. 1d) supported the similarity between the AOSD and SJIA populations.

\section{Population Model-Based Analyses}

For patients with sJIA, the predicted canakinumab exposure was similar across all age groups (children, adolescents, and older adolescents/ young adults). The predicted steady-state exposure of the AOSD population fell within the predicted exposure range of all sJIA age groups, confirming that similar exposure is achieved in patients with AOSD or sJIA using the dosing regimen of $4 \mathrm{mg} / \mathrm{kg}$ (up to maximum $300 \mathrm{mg}$ ) Q4W s.c. (ESM Fig. 2).

DAS28-CRP improvement, continuous adapted ACR response, number of active joints (percentage change from baseline), and CRP level versus categories of predicted canakinumab concentrations showed similarity across sJIA age groups and patients with AOSD (Fig. 2a-d). Exposure-response, with respect to DAS28-CRP score, suggested that no relevant value-added therapeutic benefit can be expected from a canakinumab dose increase in patients with AOSD (Fig. 3). There was no evidence of a difference in PKPD relationship between patient populations for adapted ACR30 response or absence of fever. 
A

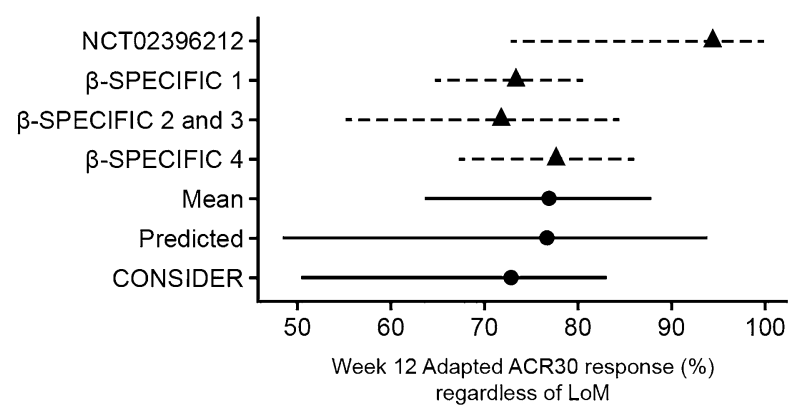

C

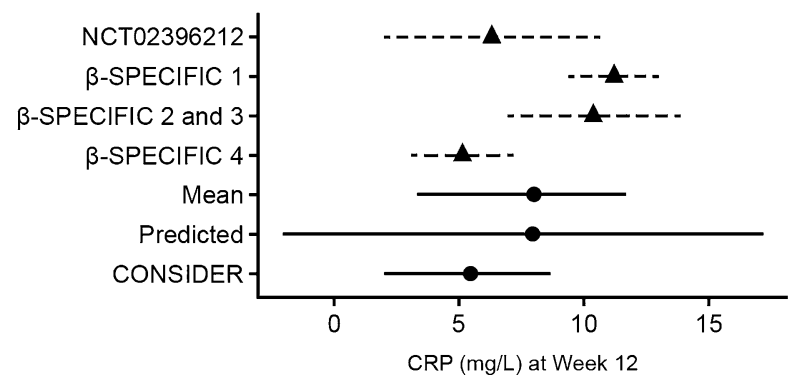

Fig. 1 Bayesian analyses of adapted ACR30 response, reduction in the number of active joints from baseline, CRP level and absence of fever at week 12 using data from the AOSD study and data borrowed from the pooled sJIA studies. a Adapted ACR30 response at week 12, b reduction of active joints at week 12, c CRP level at week 12, d absence of fever (\%) at week 12. Dashed lines refer to the mean data (filled triangles) and $95 \%$ confidence intervals for each individual study; The solid line "Mean" is the meta-analytic median (dot) for the mean response from sJIA studies and 95\% credible interval; the solid line

\section{DISCUSSION}

Bayesian analysis is a method of statistical inference that utilizes prior information derived from sources in a specific population to update the evidence for another group, particularly in clinical conditions with limited patient numbers, where relevant data from external sources are available $[10,11]$.

As it is accepted that sJIA and AOSD form the Still's disease continuum [1-3], Bayesian and population model-based analyses were considered appropriate approaches to explore similarity in clinical outcomes between the patient populations. The analyses were conducted on endpoints from studies of canakinumab in sJIA
B

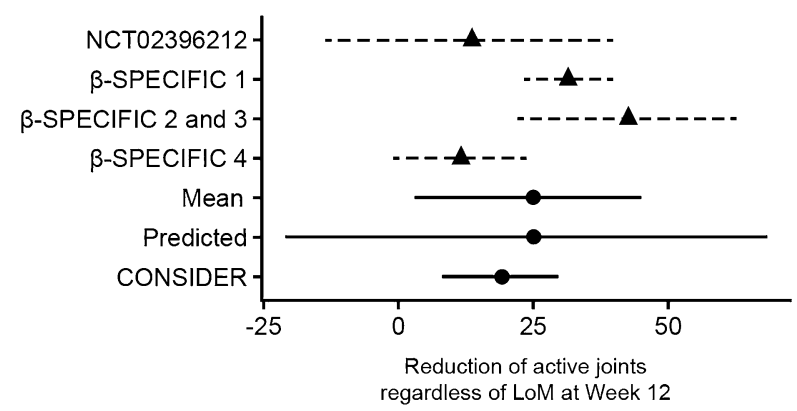

D

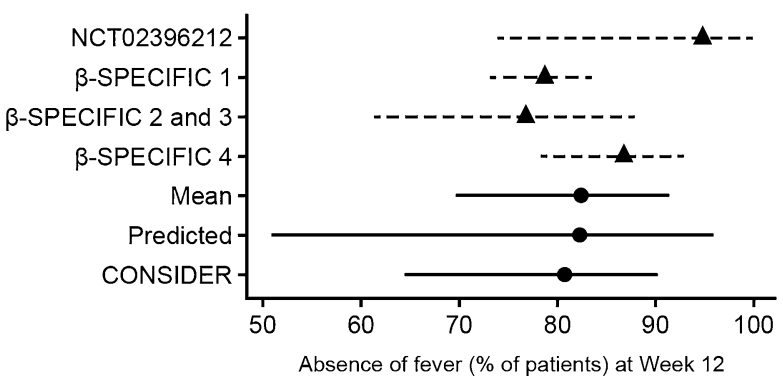

"Predicted" shows the predicted median (dot) derived from the meta-analytic predictive (MAP) prior distribution from the sJIA studies and the corresponding 95\% credible interval; The solid line "CONSIDER" shows the posterior median (dot) from the Bayesian update of the robustified meta-analytic prior from the sJIA data and $95 \%$ credible interval. $A C R$ American College of Rheumatology, AOSD adult-onset Still's disease, CRP C-reactive protein, $L o M$ limitation of movement, $s J L A$ systemic juvenile idiopathic arthritis

to support the efficacy of canakinumab in patients with AOSD, providing further evidence for the US regulatory approval in this indication. The Bayesian analysis supported similarity in clinical outcomes across patients with AOSD and sJIA following treatment with canakinumab.

The use of Bayesian approaches in the context of pediatric and adult studies requires that the smaller body mass of pediatric patients be taken into account, as well as possible differences in absorption, metabolism, and excretion of treatment between populations [11]. These aspects were addressed by population-based PK and PKPD analyses, the results of which suggested the appropriateness of the approved sJIA treatment regimen for both populations. 


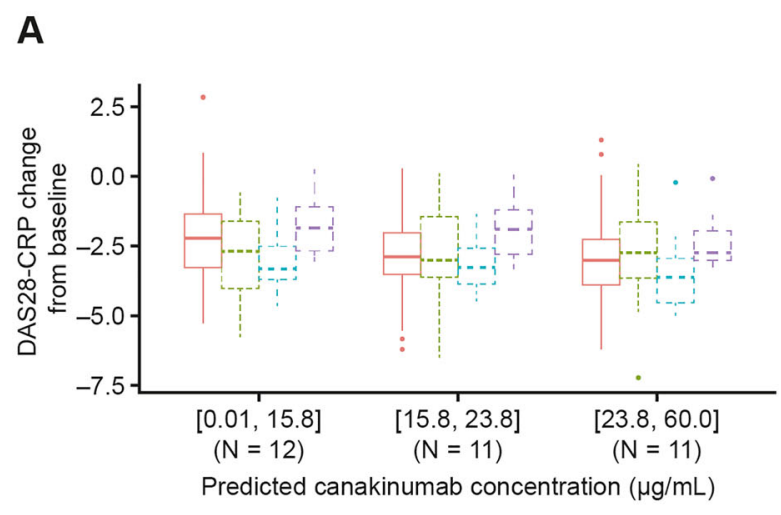

C

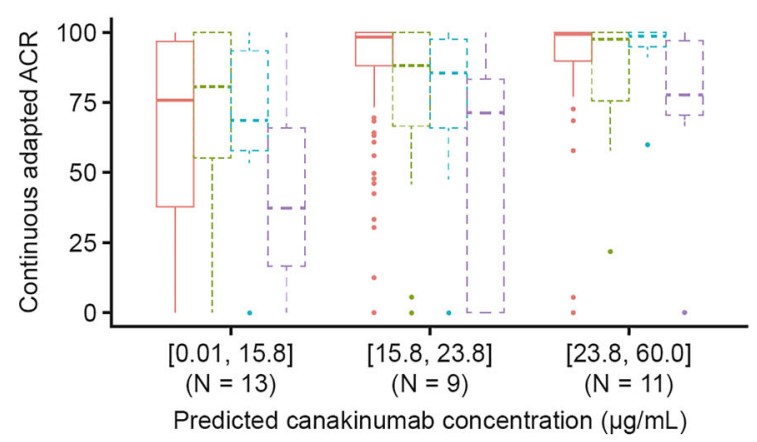

Fig. 2 Population pharmacokinetic/pharmacodynamic (PKPD) analyses between sJIA and AOSD studies. a DAS28-CRP change from baseline versus predicted canakinumab concentrations at week 8 and 12, b CRP level versus predicted canakinumab concentrations at week 8 and 12, c continuous adapted ACR response versus predicted canakinumab concentrations at week 8 and 12, d percentage change in number of active joints from baseline versus predicted canakinumab concentrations at week 8 and 12. Predicted canakinumab concentrations are

Inappropriate Bayesian extrapolation (in case of differences in distribution between the AOSD study and the MAP prior from the sJIA studies) was accounted for by robustification of the MAP prior. The robust MAP approach was described by Schmidli et al. in 2014 for the situation that historical control data are available [14]. Here, appropriate historical control data were not available from the sJIA studies. In the AOSD study, Bayesian comparisons of the augmented treatment group with the placebo group could have been performed; however, this would not have explored similarity of
B

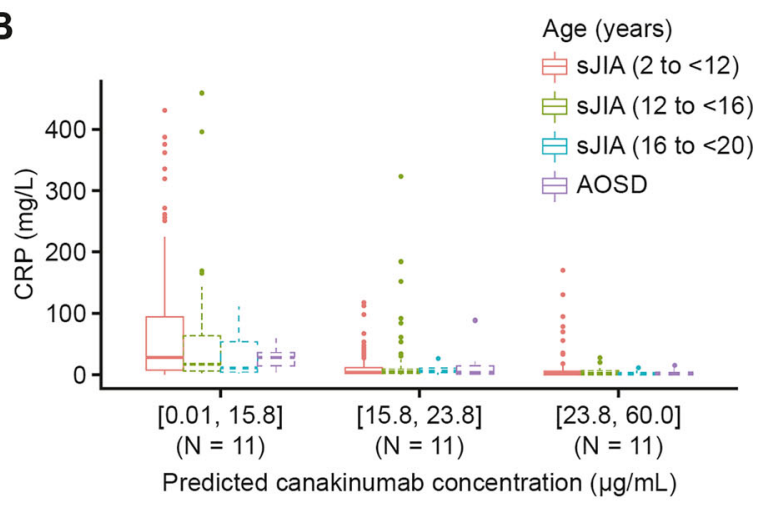

D

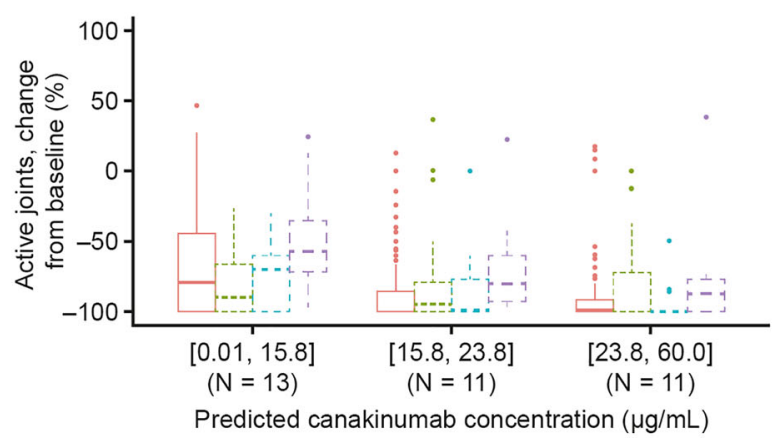

displayed by tertile exposure ranges. The lower and upper ends of boxes represent the 25th and 75th percentiles of distribution, the bold line in the box represents the median, and the whiskers extend to 1.5 -fold the interquartile range. Dots represent the outliers. $\mathrm{N}$ corresponds to the number of observations for AOSD patients in each concentration category. AOSD Adult-onset Still's disease, DAS28-CRP Disease Activity Score 28 joint count using CRP/erythrocyte sedimentation rate (DAS28[ESR/ CRP]), sJIA systemic juvenile idiopathic arthritis

clinical outcomes between the sJIA and AOSD patient populations.

The criterion for similarity of outcomes was based on the posterior medians from the AOSD study and the predicted $95 \%$ credible interval of the sJIA studies. The similarity of outcomes could have been assessed by alternative criteria, for example, a criterion by Box [16]. However, an approach which could be graphically illustrated by forest plots and 95\% credible intervals (Fig. 1) was preferred.

Within the US Food and Drug Administration, the Center for Devices and Radiologic Health have issued a formal guidance document 


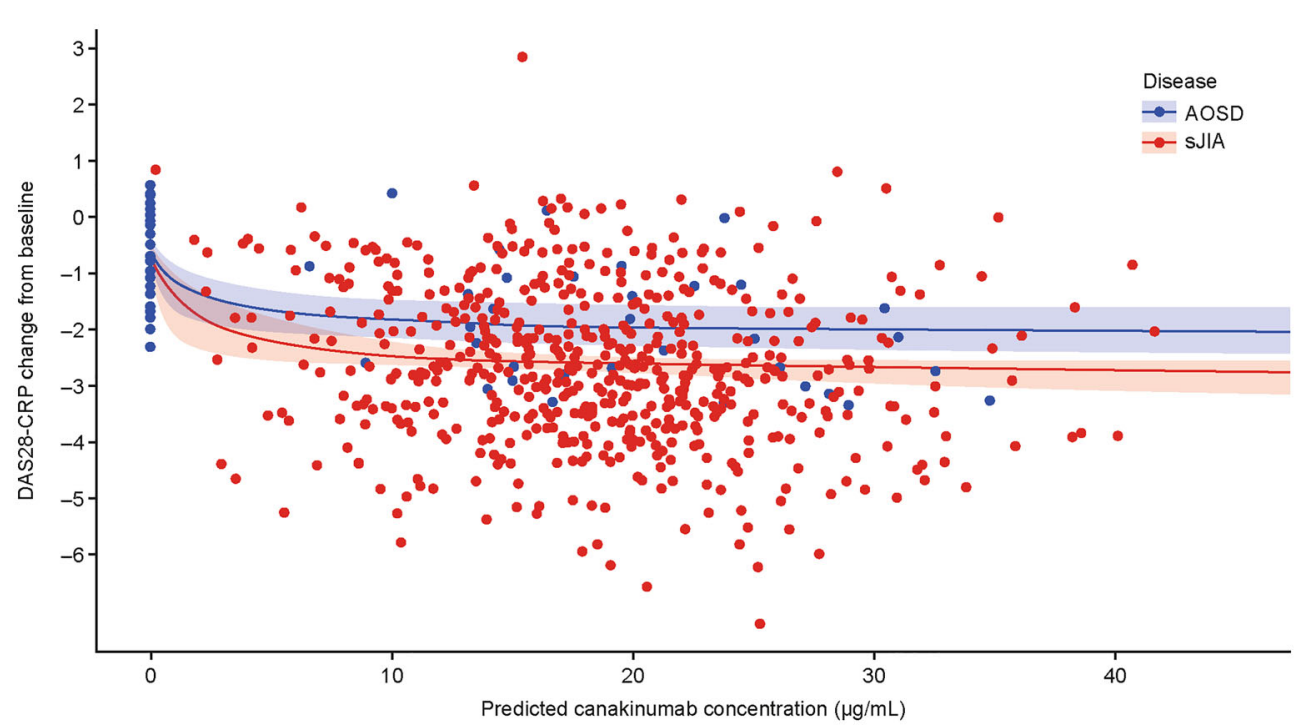

Fig. 3 Emax model fit for DAS28-CRP change from baseline versus predicted canakinumab concentrations at weeks 8 and 12 across sJIA and AOSD studies. Observed

encouraging submissions of new products using a Bayesian approach as the definitive analysis to support decision-making. Although the Center for Drug Evaluation and Research has issued no guidance on Bayesian approaches, there are several areas where Bayesian approaches are emerging, including rare diseases [11].

The results of the population PKPD analyses for binary variables (adapted ACR30 response and absence of fever) are not reported in this publication due to the nature of binary endpoints and associated small sample size, resulting in wide confidence intervals in the estimated response rates for each concentration category, making comparisons difficult. For the other endpoints, the PKPD relationships showed a slightly reduced response in patients with AOSD but were considered overall similar across age groups. These analyses also indicated that no relevant therapeutic benefit can be expected from a canakinumab dose increase in patients with AOSD.

\section{CONCLUSION}

The analyses described in this publication support the similarity of clinical outcomes data (dots) and Emax (maximal effect at high drug concentrations) model fit (solid lines) are shown with $90 \%$ confidence intervals

following treatment with canakinumab across patients with AOSD or sJIA. As it is well accepted that sJIA and AOSD are part of the Still's disease continuum, the results presented here are clinically meaningful and further support the efficacy of canakinumab in patients with AOSD.

\section{ACKNOWLEDGEMENTS}

Funding. Financial support for the writing and publication of this paper was provided by Novartis, including the Rapid Service Fee.

Medical Writing Assistance. Medical writing support for the development of this manuscript, under the direction of the authors, was provided by Samuel McCracken, of Ashfield MedComms, an Ashfield Health company, and funded by Novartis Pharma AG.

Authorship. All named authors meet the International Committee of Medical Journal Editors (ICMJE) criteria for authorship for this article, take responsibility for the integrity of 
the work as a whole, and have given their approval for this version to be published.

Author Contributions. All authors were involved in drafting the article or revising it critically for important intellectual content, and all authors approved the final version to be published. $\mathrm{CDB}, \mathrm{SN}, \mathrm{SW}$, and $\mathrm{BB}$ were involved in the acquisition of the data. $\mathrm{CDB}$ and $\mathrm{BB}$ were involved in the analysis of the data. $\mathrm{CDB}, \mathrm{SN}$, SW, BB were involved in the interpretation of the data.

Prior Presentation. The studies used for these analyses have previously been published (details outlined in references).

Disclosures. Cornelia Dunger-Baldauf, Priscila Nakasato, Stephanie Noviello, Sarah Whelan, and Bruno Bieth are employees of Novartis Pharma AG or Novartis Pharmaceuticals Corporation.

Compliance with Ethics Guidelines. This article is based on previously conducted studies and does not contain any new studies with human participants or animals performed by any of the authors. The ethics approvals for the studies outlined have been detailed previously in their respective publications.

Data Availability. All data generated or analyzed during this study are included in this published article and its supplementary information files.

Open Access. This article is licensed under a Creative Commons Attribution-NonCommercial 4.0 International License, which permits any non-commercial use, sharing, adaptation, distribution and reproduction in any medium or format, as long as you give appropriate credit to the original author(s) and the source, provide a link to the Creative Commons licence, and indicate if changes were made. The images or other third party material in this article are included in the article's Creative Commons licence, unless indicated otherwise in a credit line to the material. If material is not included in the article's Creative Commons licence and your intended use is not permitted by statutory regulation or exceeds the permitted use, you will need to obtain permission directly from the copyright holder. To view a copy of this licence, visit http://creativecommons.org/licenses/by$\mathrm{nc} / 4.0 /$.

\section{REFERENCES}

1. Nirmala N, Brachat A, Feist E, et al. Gene-expression analysis of adult-onset Still's disease and systemic juvenile idiopathic arthritis is consistent with a continuum of a single disease entity. Pediatr Rheumatol Online J. 2015;13:50.

2. Feist E, Quartier P, Fautrel B, et al. Efficacy and safety of canakinumab in patients with Still's disease: exposure-response analysis of pooled systemic juvenile idiopathic arthritis data by age groups. Clin Exp Rheumatol. 2018;36:668-75.

3. Jamilloux Y, Gerfaud-Valentin M, Martinon F, Belot A, Henry T, Seve P. Pathogenesis of adult-onset Still's disease: new insights from the juvenile counterpart. Immunol Res. 2015;61:53-62.

4. Kedor C, Listing J, Zernicke J, et al. Canakinumab for Treatment of Adult-Onset Still's Disease to Achieve Reduction of Arthritic Manifestation (CONSIDER): phase II, randomised, double-blind, placebo-controlled, multicentre, investigator-initiated trial. Ann Rheum Dis. 2020;79:1090-7.

5. Vastert SJ, Jamilloux Y, Quartier P, et al. Anakinra in children and adults with Still's disease. Rheumatology (Oxford). 2019;58:vi9-22.

6. Ruperto N, Brunner HI, Quartier P, et al. Two randomized trials of canakinumab in systemic juvenile idiopathic arthritis. N Engl J Med. 2012;367: 2396-406.

7. Ruperto N, Brunner HI, Quartier P, et al. Canakinumab in patients with systemic juvenile idiopathic arthritis and active systemic features: results from the 5-year long-term extension of the phase III pivotal trials. Ann Rheum Dis. 2018;77:1710-9.

8. Nishimura K, Hara R, Umebayashi H, et al. Efficacy and safety of canakinumab in systemic juvenile idiopathic arthritis: 48-week results from an openlabel phase III study in Japanese patients. Mod Rheumatol. 2021;31:226-34.

9. Quartier P, Alexeeva E, Constantin T, et al. Tapering canakinumab monotherapy in patients with systemic juvenile idiopathic arthritis in clinical 
remission: results from a phase IIIb/IV open-label, randomized study. Arthritis Rheumatol. 2021;73: 336-46.

10. Schmidli H, Häring D, Thomas M, Cassidy A, Weber S, Bretz F. Beyond randomized clinical trials: use of external controls. Clin Pharmacol Ther. 2020;107: 806-16.

11. Ruberg S, Harrell F, Gamalo-Siebers M, et al. Inference and decision making for 21st-century drug development and approval. Am Stat. 2019;73: 319-27.

12. Food And Drug Administration (FDA). Population Pharmacokinetics Draft Guidance for Industry 2019. https://www.fda.gov/media/128793/ download. Accessed June 2021.

13. European Medicines Agency (EMA). ILARIS (canakinumab): European Public Assessment Report
(EPAR).

https://www.ema.europa.eu/en/ documents/variation-report/ilaris-h-c-1109-ii-0026epar-assessment-report-variation_en.pdf. Accessed June 2021.

14. Schmidli H, Gsteiger S, Roychoudhry S, O'Hagan A, Spiegelhalter D, Neuenschwander B. Robust metaanalytic-predictive priors in clinical trials with historical control information. Biometrics. 2014;70: 1023-32.

15. Chakraborty A, Tannenbaum S, Rordorf C, et al. Pharmacokinetic and pharmacodynamic properties of canakinumab, a human anti-interleukin-1beta monoclonal antibody. Clin Pharmacokinet. 2012;51:e1-18.

16. Box E. Sampling and Bayes inference in scientific modeling and robustness. J R Stat Soc Ser A. 1980;143:383-430. 